\title{
Multiparameter correlation microscopy of blood plasma polycrystalline networks in the diagnosis of cancer tissues of female reproductive system
}

\author{
Koval G.D., Balazyuk V.N., Sidor M.I. \\ Correlation Optics Department, \\ Chernivtsi National University, \\ 2 Kotsyubinsky Str., Chernivtsi, 58012, Ukraine \\ yuriyu@gmail.com
}

\begin{abstract}
The principles of optical model of human bile polycrystalline structure are described. The results of investigating the interrelation between the values of statistical, correlation and fractal parameters are presented. They characterize the coordinate distributions of mutual polarization degree of the points of laser images of bile smears of cholelithiasis patients in combination with other pathologies. The diagnostic criteria of the cholelithiasis nascency and its severity degree differentiation are determined.
\end{abstract}

Key words: laser polarimetry, optical diagnostic, plasma, mutual polarization, polarization selection, polycrystalline structure

\section{Introduction}

Among the methods of optical diagnostics of human biological tissues the techniques of laser polarimetry diagnostics of their optical anisotropic structure became widely spread [1-15].

The main information for these methods is obtained from coordinate distributions of polarization azimuths $\alpha(x, y)$ and ellipticity $\beta(x, y)$ (polarization maps) with the following correlation (auto- and mutually correlation functions and fractal (fractal dimensions analysis [6,7].

As a result, several techniques of early diagnostics and differentiation of pathological changes in biological tissue (BT) structure with their degenerative, dystrophic and oncological changes were developed [8-11].

Besides, there is a widely spread group of optically anisotropic biological objects, for which the techniques of laser polarimetry diagnostics are not efficient enough. Optically thin (attenuation coefficient $\tau \leq 0,1$ ) layers of different biological fluids (bile, urine, liquor, synovial fluid, blood plasma, etc.) belong to such objects. Biological fluids are much more accessible for direct laboratory analysis if compared with traumatic techniques of the BT biopsy.

In terms of the above mentioned the task of searching new additional parameters for laser diagnostics of biological fluids' optical anisotropic structure appears to be topical.

This research is focused on the analysis of potentiality of diagnostics and differentiation of cholelithiasis of patients with chronic cholecystitis and $2^{\text {nd }}$ type diabetes by means of new technique of polarization correlometry of human plasma layers laser images.

2. The Technique of Coordinate Distribution of Mutual Polarization Complex Degree procedure:

The technique of determining the parameter of mutual polarization complex degree consists in the following 
1. By rotating the transmission plane of polarizer within the rotation angle $\theta 0^{0}-180^{\circ}$ the arrays of minimal and maximal intensity levels $I_{\min }\left(\begin{array}{l}r_{11}, \ldots r_{1 m} \\ \ldots \ldots \ldots \\ r_{n 1}, \ldots r_{n m}\end{array}\right) ; I_{\max }\left(\begin{array}{l}r_{11}, \ldots r_{1 m} \\ \ldots \ldots \ldots \\ r_{n 1}, \ldots r_{n m}\end{array}\right)$ of human bile layers images for each separate pixel $(\mathrm{mn})$ of CCD-camera were determined, as well as rotation angles $\left.\theta\left(\begin{array}{l}r_{11}, \ldots r_{1 m} \\ \ldots \ldots \ldots \\ r_{n 1}, \ldots . . .\end{array}\right)\left(\begin{array}{l}r_{n m}\end{array}\right)\left(\begin{array}{l}r_{11}, \ldots r_{1 m} \\ \ldots \ldots \ldots . . \\ r_{n 1}, \ldots . r_{n m}\end{array}\right) \equiv \min \right)$ corresponding to them.

2. The coordinate distributions (polarization maps) of polarization states in the plane of human bile samples images were calculated by such relations

$$
\begin{aligned}
\alpha\left(\begin{array}{l}
r_{11}, \ldots r_{1 m} \\
\ldots \ldots \ldots . . . \\
r_{n 1}, \ldots r_{n m}
\end{array}\right) & =\theta\left(I\left(r_{i}\right) \equiv \min \right)-\frac{\pi}{2} \\
\beta\left(\begin{array}{l}
r_{11}, \ldots . r_{1 m} \\
\ldots \ldots \ldots . . \\
r_{n 1}, \ldots r_{n m}
\end{array}\right) & =\operatorname{arctg} \frac{I\left(r_{i}\right)_{\min }}{I\left(r_{i}\right)_{\max }} .
\end{aligned}
$$

1. The value of complex degree of mutual polarization $V(r ; r+\Delta r)$ of human bile samples' laser images was calculated by the following relation

$$
V(r ; r+\Delta r)=\frac{2\left\{I_{0} I_{90} \cos [\arcsin (\cos 2 \alpha / \operatorname{tg} 2 \beta)]\right\}(r) \times\left\{I_{0} I_{90} \cos [\arcsin (\cos 2 \alpha / \operatorname{tg} 2 \beta)]\right\}(r+\Delta r)}{\left(I_{0}^{2}(r)+I_{90}^{2}(r)\right)\left(I_{0}^{2}(r+\Delta r)+I_{90}^{2}(r+\Delta r)\right)} .
$$

\section{Experimental Results and Discussion}

Laser images of three groups of blood plasma samples of the patients of different pathological state:

- healthy patients - group 1 (11 patients);

- patients with endometrioses - group 2 (10 patients);

- cancer patients - group 3 (12 patients).

The coordinate distribution and histogram of random values of $V(x, y)$ parameter of polarizationallyinhomogeneous laser image of blood plasma layer laser image of a healthy patient are presented in Fig. 1.

It can be seen from the obtained data that the laser image of a healthy patient's bile layer is characterized with a high homogeneity of polarization parameters - the number of values $V(x, y)=1$ is by three orders higher than the other, non-zero values of mutual polarization degree.

In other words, in biochemical structure of this bile layer the optically isotropic component prevails. 

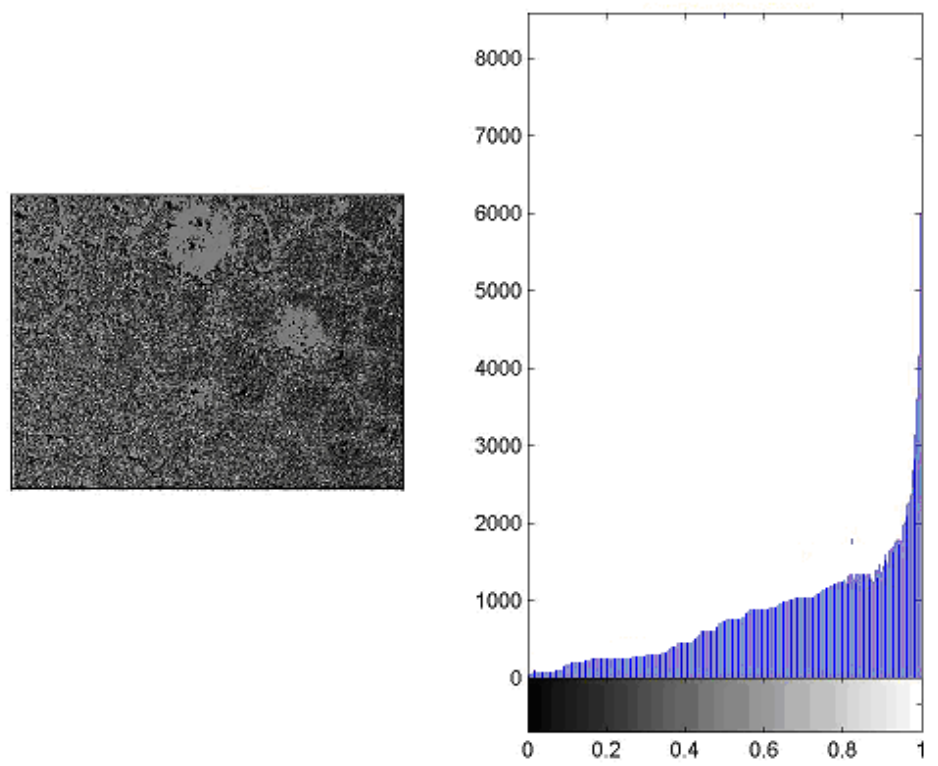

Figure 1. Coordinate distribution (a) and histogram of values (a) of mutual polarization $V(x, y)$ degree if a healthy patient's blood plasma layer (group 1).

Correlation (b) fractal (c), structure of distribution (a) and the amount (b) of values of parameter $V(x, y)=0,5$ characterizing the liquid crystalline component of blood plasma of patients from group 1 are presented in Fig. 2.

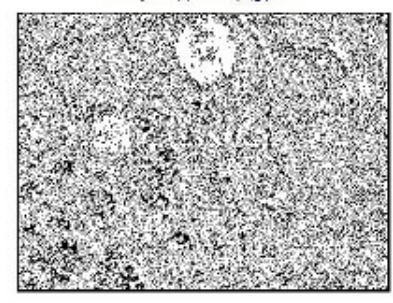

(a)

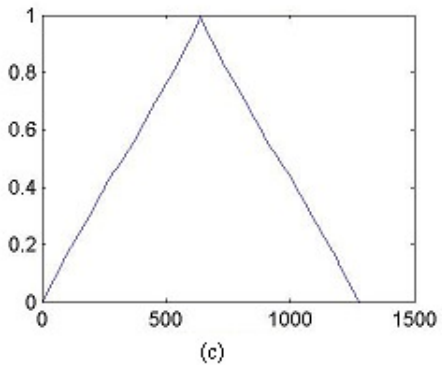

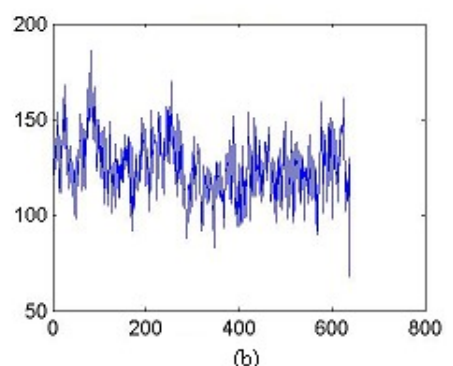

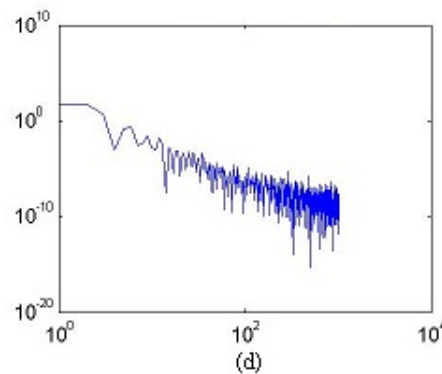

Figure 2. Autocorrelation function (c) and $\log$-log dependencies (d) of the amount of values $V=0,5$ (b) in the coordinate distribution $V(x, y)$ (a) of a healthy patient's blood plasma layer (group 1).

It was determined that the set of values $V(x, y)=0,5$ is fractal $(D(V=0,5)=2,11 ; \Omega(V=0,5)=0,16)$ with correlation area $S(V=0,5)=0,16$ great enough.

For biochemical composition of blood plasma layer from group 2 a much more developed optically anisotropic component is typical - Fig. 3 . 
It can be seen from the analysis of histograms of random values of mutual polarization degree of the laser image of blood plasma layer of chronic cholecystitis patient that the number of values $V(x, y)=0,5$ (liquid crystalline fraction) amount to $15 \%$ of the number of values $V(x, y)=1,0$ characterizing the images of optically isotropic component.

The corresponding statistical (b), correlation (c) and fractal (d) parameters of coordinate distributions $V=0,5$ (a) are presented in Fig.4.
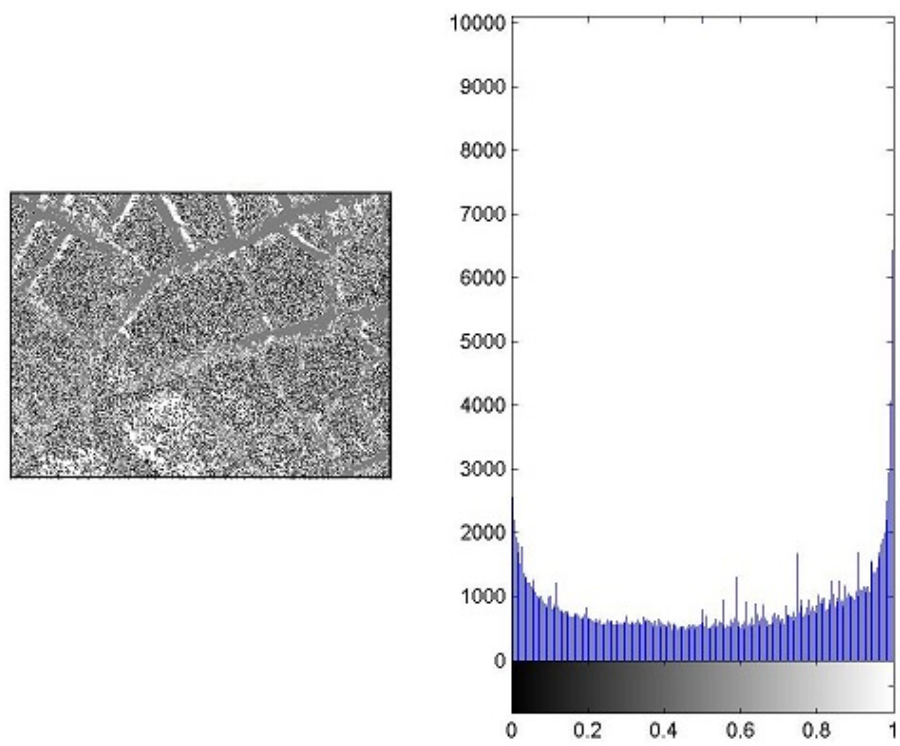

Figure 3. Coordinate distribution (a) and histogram of the values (a) of mutual polarization degree $V(x, y)$ of blood plasma layer of group 2.

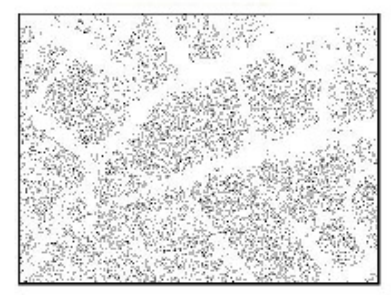

(a)

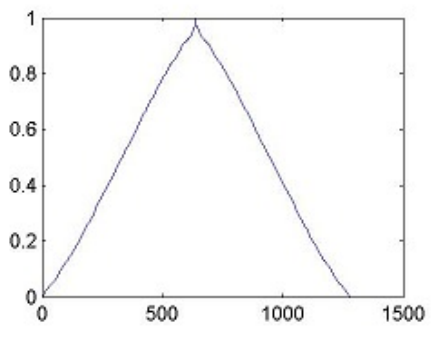

(c)

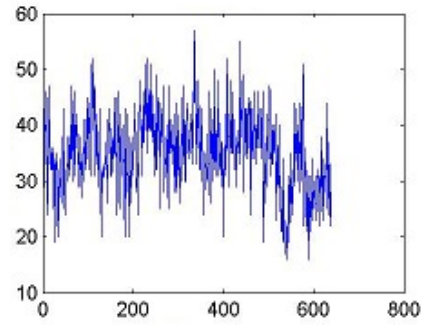

(b)

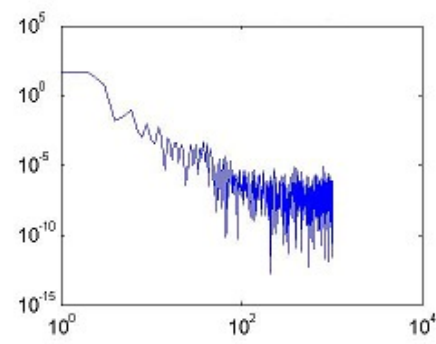

(d)

Figure 4. Autocorrelation function (c) and $\log -\log$ dependencies (d) of the amount of values $V=0,5$ (b) in the coordinate distribution $V(x, y)$ (a) of blood plasma layer of group 2.

It was determined for liquid crystalline fraction of blood plasma layer that the set of values $V(x, y)=0,5$ is fractal $(D(V=0,5)=2,03 ; \Omega(V=0,5)=0,21)$ with maximally great correlation area $S(V=0,5)=0,24$. 
The following peculiarities are typical for polarization-correlation structure of laser images of bile layers of second type diabetes patients (Fig. 5, Fig. 6).
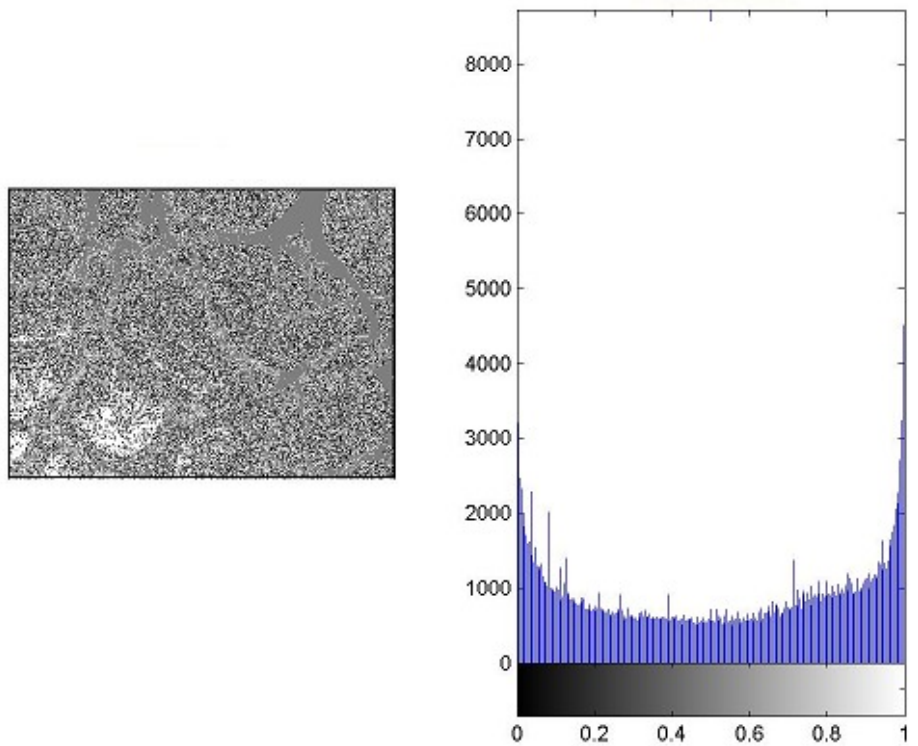

Figure 5. Coordinate distribution (a) and histogram (a) of the values of mutual polarization degree $V(x, y)$ of blood plasma layer of group 3.

The extreme values of distribution $V(x, y)$ of blood plasma layer of a patient from group 3, corresponding to sampling $V=0,5$, increase and amount to $45 \%-50 \%$.

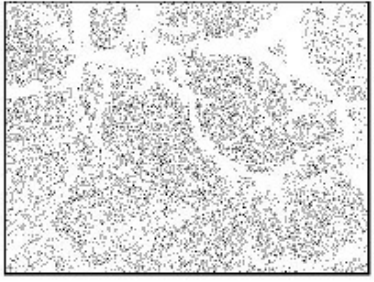

(a)

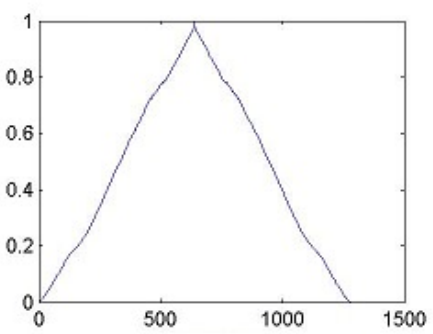

(c)

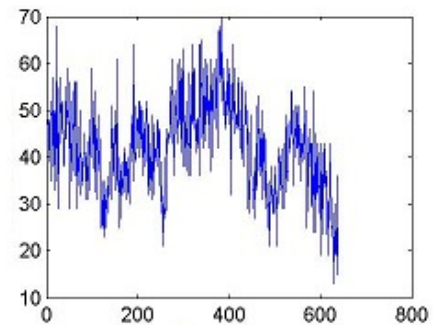

(b)

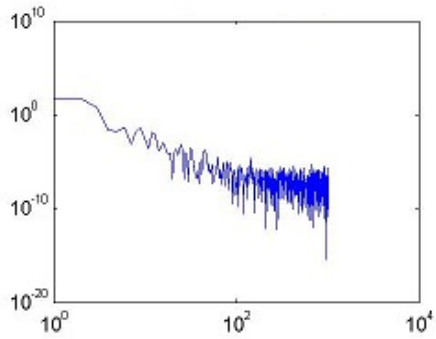

(d)

Figure 6. Autocorrelation function (c) and $\log$-log dependencies (d) of the amount of values $V=0,5$ (b) in the distribution $V(x, y)$ (a) of blood plasma layer of group 3 .

The correlation area and dispersion of extremes distribution of log-log dependency of power spectra of the number of extreme values of mutual polarization degree $V=0,5$ of the laser image of blood plasma layer of the patient with complex pathology are as follows: $S(V=0,5)=0,25$ and $D(V=0,5)=1,93 ; \Omega(V=0,5)=0,29$. 


\section{Criteria of Diagnostics of the Patients with Different Types of Pathology}

The following parameters of values distribution of liquid-crystalline sampling $(V(x, y)=0,5)$ of mutual polarization degree of laser images of human blood plasma layers belong to the basic criteria of diagnosing:

- statistical moments $\left(M_{i=1 ; 2 ; 3 ; 4}(V)\right)$ of distribution of mutual polarization degree values $V(x, y)=0,5$;

- correlation areas $S(V=0,5)$ of distribution of mutual polarization degree values $V(x, y)=0,5$;

- dispersions $\Omega(V)$ of extremes distribution of log-log dependencies of power spectra of parameters $V(x, y)=0,5$ values.

The ensemble of data about the values of diagnostic parameters $M_{k=1 ; 2 ; 3 ; 4}(V=0,5)$ is presented in Table 1.

Table 1. Statistical moments of the $1^{\text {st }}-4^{\text {th }}$ orders of distributions $V(x, y)=0,5$ of bile layers of all groups of patients

\begin{tabular}{|c|c|c|c|}
\hline Parameters & Group 1 & Group 2 & Group 3 \\
\hline$M_{1}(V=0,5)$ & 0,09 & 0,21 & 0,32 \\
& \pm & \pm & \pm \\
& 0,008 & 0,027 & 0,019 \\
\hline$M_{2}(W=0,5)$ & 0,26 & 0,13 & 0,12 \\
& \pm & \pm & \pm \\
& 0,031 & 0,023 & 0,019 \\
\hline$M_{3}(W=0,5)$ & 0,11 & 1,28 & 4,26 \\
& \pm & \pm & \pm \\
& 0,021 & 0,41 & 0,58 \\
\hline$M_{4}(W=0,5)$ & \pm & 2,12 & 5,29 \\
& 0,009 & \pm & \pm \\
& & 0,52 & 0,0096 \\
\hline
\end{tabular}

The obtained data about the coordinate distributions of mutual polarization degree of laser images of blood plasma of all groups of healthy and sick patients prove that the statistical analysis of dependencies of the number of values of $V(x, y)=0,5$ sampling (liquid-crystalline phase) of blood plasma layers laser images.

The difference between statistical moments $M_{k}(W)$ of laser images of test group patients' blood plasma (group 1) and the patients with various pathologies (groups 2 and 3) - mean (increasing by $2.7-3.5$ times); dispersion (decreasing by 2.5 - 3.3 times); asymmetry (increasing by $3.3-5.4$ times) and excess (increasing by $4.5-6.1$ times) - are determined.

Thus, it can be stated that statistical moments' $M_{k=1 ; 2 ; 3 ; 4}(V=0,5)$ investigation enables to perform reliable differentiation of the patients from groups 2 and 3.

Comparative data of correlation and fractal parameters of extreme values $V(x, y)=0,5$ distribution of laser images of all groups of patients are presented in Table 2.

Table 2. Correlation $(S(V=0,5))$ and fractal $(\Omega(V=0,5))$ parameters of $V(x, y)=0,5$ distributions of blood plasma layers of all groups of patients

\begin{tabular}{|c|c|c|c|}
\hline Parameters & Group 1 & Group 2 & Group 3 \\
\hline$S(V=0,5)$ & 0,15 & 0,22 & 0,29 \\
& \pm & \pm & \pm \\
& 0,038 & 0,042 & 0,036 \\
\hline$\Omega(V=0,5)$ & 0,17 & 0,24 & 0,38 \\
& \pm & \pm & \pm \\
& 0,048 & 0,069 & 0,089 \\
\hline
\end{tabular}

The data about correlation and fractal structure of extreme values $V(x, y)=0,5$ distributions of mutual polarization degree indicate that the value of correlation area $S(V=0,5)$ and power spectra dispersion $\Omega(V=0,5)$ of mutual polarization degree distributions enable to reliably diagnose with different pathology types. Correlation area $S(V=0,5)$ increases by 1.7-1.9 times. Dispersion $\Omega(V=0,5)$ increases by 1.6-2.1 times. 


\section{Conclusions}

1. A new technique of estimating the structure of laser images based on measuring coordinate distributions of mutual polarization degree is suggested that characterizes the homogeneity of optically isotropic and optically anisotropic components in biochemical composition of blood plasma.

2. The statistical (mean, dispersion, asymmetry and excess), correlation (correlation area of distribution of mutual polarization degree values) and fractal (dispersion of extremes of log-log dependencies of power spectra of mutual polarization degree values distribution) criteria of polarization-correlation diagnostics all groups blood plasma layers.

\section{References}

[1] Handbook of Optical Coherence Tomography; edited by B.E. Bouma and G.J. Tearney // Polarization-sensitive optical coherence tomography / J. F. de Boer, T. E. Milner, M. G. Ducros, S. M. Srinivas and J. S. Nelson. Marcel Dekker Inc.: New York, 2002. - P. 237-274.

[2] Everett, M. J., Shoenenberger, K., Colston, B. W., Da Silva, L. B., "Birefringence characterization of biological tissue by use of optical coherence tomography", Opt. Lett., Vol. 23., P. 228-230, (1998).

[3] Ducros, M. G., de Boer, J. F., Huang, H. E., Chao, L. C., Chen, Z. P., Nelson, J. S., Milner, T. E., Rylander, H. G., "Polarization sensitive optical coherence tomography of the rabbit eye", IEEE J. Select. Top. Quant. Electron, Vol. 5, P. 1159-1167 (1999).

[4] de Boer, J. F., Milner, T. E., van Gemert, M. J., Nelson, J. S., John, S., "Two-dimensional birefringence imaging in biological tissue using polarization-sensitive optical coherence tomography", Proc. SPIE., Vol. 3196, P. 32-37 (1998).

[5] de Boer, J. F., Milner, T. E., Nelson, J. S., "Determination of the depth-resolved Stokes parameters of light backscattered from turbid media by use of polarization-sensitive optical coherence tomography", Opt. Lett., Vol. 24, P. 300-302 (1999).

[6] Ostermeyer, M. R., Stephens, D. V., Wang, L. and Jacques, S. L., "Nearfield Polarization Effects on Light Propagation in Random Media", Trends in Optics and Photonics: Biomedical Optical Spectroscopy and Diagnostics, 3, 20-26 (1996).

[7] Bruscaglioni, A., Zaccanti, G., Wei, Q.,"Transmission of a pulsed polarized light beam through thick turbid media: numerical results", Appl. Opt., 32, 6142- 6150 (1993).

[8] V. Sankaran, M. J. Everett, D. J. Maitland, J. T. Walsh, "Comparison of polarized-light propagation in biological tissue and phantoms", Opt. Lett, 24, 1044-1046 (1999).

[9] Angelsky, O.V., Ushenko, A.G., Ushenko, Yu.A., Ushenko, Ye.G., "Polarization singularities of the object field of skin surface", Journal of Physics D: Applied Physics, 39 (16), art. no. 005, pp. 3547-3558 (2006).

[10] Angelsky, O.V., Demianovsky, G.V., Ushenko, A.G., Burkovets, D.N., Ushenko, Yu.A., "Wavelet analysis of two-dimensional birefringence images of architectonics in biotissues for diagnosing pathological changes", Journal of Biomedical Optics, 9 (4), pp. 679-690. (2004)

[11] Angelsky, O.V., Ushenko, A.G., Arkhelyuk, A.D., Ermolenko, S.B., Burkovets, D.N., Ushenko, Yu.A., "Laser Polarimetry of Pathological Changes in Biotissues", Optics and Spectroscopy, 89 (6), pp. 973-978, (2000).

[12] Angelsky, O. V., Bekshaev, A. Ya., Maksimyak, P. P., Maksimyak, A. P.; Hanson, S. G.; Zenkova, C.Yu., "Orbital rotation without orbital angular momentum: mechanical action of the spin part of the internal energy flow in light beams", Optics Express, Vol. 20 Issue 4, pp.3563-3571,(2012).

[13] Angelsky, O. V., Bekshaev, A. Ya., Maksimyak, P. P., Maksimyak, A. P., Mokhun, I. I., Hanson, S. G., Zenkova, C. Yu., Tyurin, A. V., "Circular motion of particles suspended in a Gaussian beam with circular polarization validates the spin part of the internal energy flow", Optics Express, Vol. 20 Issue 10, pp.1135111356,(2012).

[14] A. Y. Bekshaev, O. V. Angelsky, S. G. Hanson, and C. Y. Zenkova, "Scattering of inhomogeneous circularly polarized optical field and mechanical manifestation of the internal energy flows", Phys. Rev. A 86, 023847, (2012).

[15] O. V. Angelsky, A. Ya. Bekshaev, P. P. Maksimyak, A. P. Maksimyak, S. G. Hanson, and C. Yu. Zenkova, "Self-diffraction of continuous laser radiation in a disperse medium with absorbing particles", Optics Express, Vol. 21, Issue 7, pp. 8922-8938, (2013). 